\section{Protection from periodontitis}

Help patients manage the effects of periodontal disease with a naturally powerful mouth rinse. The Perio Plus+ product range offers both short- and longer-term solutions that are designed as a useful temporary adjunct to the mechanical removal of plaque.

Perio Plus+ products combine clinically-proven chlorhexidine (CHX) with CITROX - a natural anti-microbial, anti-oxidant and anti-inflammatory substance created from the bioflavonoids found in bitter oranges. This unique formula can effectively combat plaque,
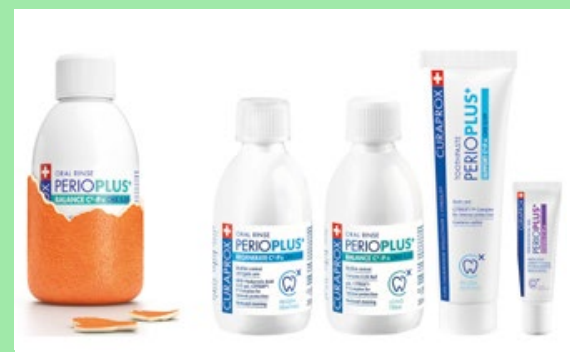
with minimal risk of adverse side effects traditionally associated with leading $\mathrm{CHX}$ mouth rinses.

For natural protection against oral diseases such as periodontitis, recommend Perio Plus+ to your patients. Order by contacting 01480 862084, emailing info@curaprox.co.uk or visiting www.curaprox.co.uk.

\title{
Arthroscopic treatment of TMJ Disorders
}

Where patients require mid-line treatment for TMJ disorders, finding the most minimally invasive solution is important. Incito Medtech offers Arthroscope Systems for this reason. Using only the highest quality of materials with

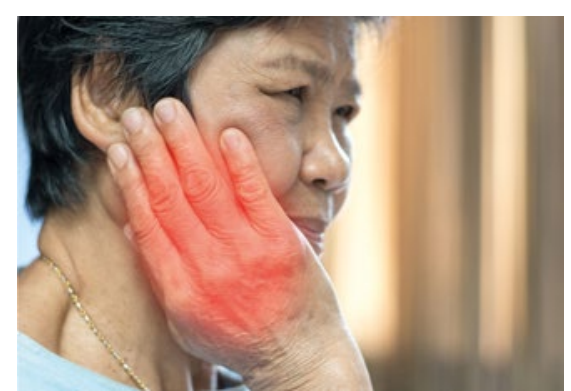

instruments that have been carefully designed for both surgical efficiency and patient comfort, the systems provide the tools professionals need to ensure safe and effective arthroscopic surgery.

TMJ disorders can have a massive impact on patients' lives. Where appropriate, offering as conservative a surgery as possible to correct their problems will encourage treatment acceptance and ensure they get the care they need.

For more information about the Arthroscope Systems, call Karen Joy on 07468420496 or email Karen.Joy@ incitomedtech.co.uk or visit http:// incitomedtech.co.uk.

\section{Stay compliant}

Effective infection control demands complete compliance with various regulations. So why not choose an autoclave designed to facilitate compliance at every step?

The Little Sister SES 2020N autoclave from Eschmann keeps compliance fuss-free with a number of innovative features. Not only has it been built to the latest EN13060 standards, but it also has a built-in level of user verification and protection that meets all the latest requirements for validation and compliance.

The optional AutoLog cycle manager also helps you keep on top of compliance by giving you the ability to automate your daily and weekly validation tests!

For more information on the highly effective and affordable range of decontamination equipment and products from Eschmann, visit www.eschmann.co.uk or call 01903875787.

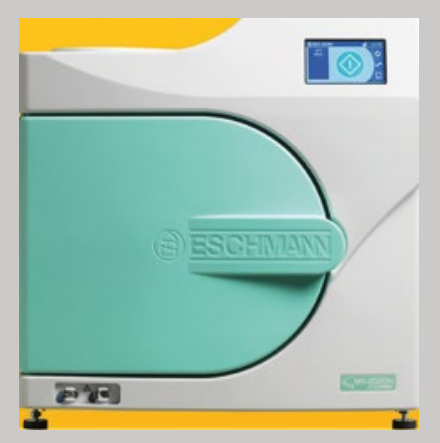

\section{Protection that works!}

Winter illnesses can be easily spread in populated areas such as waiting rooms in your practice.

Make sure that you keep patients safe from infection by using cleaning products that work, such as the Steri-7 Xtra range of disinfectants from Initial Medical.

Available in a selection of surface cleaners, hand washes and hand rubs, these innovative products deactivate 99.9999\% of all pathogens, including those responsible for the majority of winter illnesses.

Furthermore, these products have

Reactive Barrier Technology which forms a protective layer on surfaces that lasts for up to 72 hours. This means pathogens cannot recolonise in these areas during that time.

Protect your practice by contacting Initial Medical on www.initial.co.uk/ medical or calling 08708504045 .

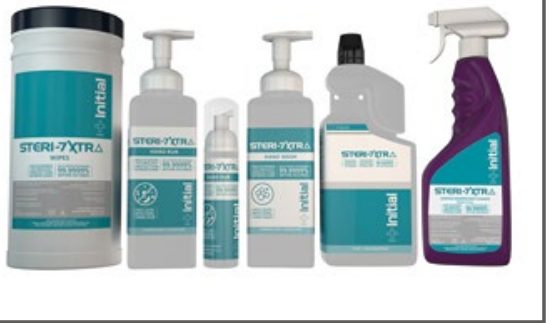

\section{Don't let dental anxiety} impact their care

The majority of the UK population suffer from dental anxieties or phobias. So why not help anxious patients receive the care they need by referring them to EndoCare?

Our highly experienced team are all highly trained in providing a supportive, calming environment for heavily anxious and phobic patients. Furthermore, as we allow patients to watch films, listen to music and provide as pain-free surgery as possible, this helps to combat the anxieties going forward, meaning that they can receive care without the fear factor.

Once we have treated your patient, we will return them to your care with all of the relevant details and aftercare suggestions.

For further information call EndoCare on 02072240999 or visit www.endocare.co.uk. 